\title{
Power Control and Beamformer Design for the Optimization of Full-Duplex MIMO Relays in a Dual-Hop MISO Link
}

\author{
Umut Ugurlu, Risto Wichman, and Taneli Riihonen \\ Aalto University School of Electrical Engineering \\ P.O. Box 13000, FI-00076 Aalto, Finland \\ Email: firstname.surname@aalto.fi
}

\author{
Cássio Ribeiro and Carl Wijting \\ Nokia Research Center \\ P.O. Box 226, FI-00045 NOKIA GROUP, Finland \\ Email: firstname.surname@nokia.com
}

\begin{abstract}
This paper studies the optimization of a dual-hop multi-input single-output (MISO) link with a full-duplex multiinput multi-output (MIMO) relay connecting the end nodes based on the amplify-and-forward or decode-and-forward protocol. The direct end-to-end link is considered weak, hence interpreted as interference by the dual-hop relay link. We observe a duality between the first and second hops in terms of transmit powers, and show that one of them always optimally converges to its upper bound. In addition, we combine the minimum mean square error-based receive beamformer with the optimal transmit powers as an iterative approach is not guaranteed to converge to global optimum. Moreover, we introduce a new figure of merit to approximate the optimal transmit beamformers, which is based on the maximization of the signal-to-leakage-plus-noise ratio, and present an iterative algorithm to jointly optimize the receive and transmit beamformers as well as the transmit powers. The numerical results demonstrate that the joint iterative algorithm can achieve higher data rates with the leakage-based transmit beamformers than those with conventional transmit filtering.
\end{abstract}

\section{INTRODUCTION}

Full-duplex systems can perform simultaneous transmission and reception in the same frequency band, hence avoiding the rate loss inherent to conventional resource division-based duplex modes [1]. Therefore, full-duplex systems can promise up to twice the data rate offered by spectral or temporal division-based duplex systems in link level.

A promising application of full-duplex mode is relaying. The most common relay strategies are amplify-andforward (AF) and decode-and-forward (DF), both of which are considered in this paper. The AF protocol requires less computations, whereas the DF protocol is more effective under low signal-to-noise ratio (SNR) conditions. The transmitted signal from a full-duplex relay is unintentionally received by its own receiver. This self-interference signal can be partly mitigated by physical isolation between the transmit and receive antennas [2]. However, the residual self-interference may still severely degrade the achievable transmission rates. The optimal transmit power control and beamformer design can combat the residual self-interference, hence achieving higher data rates.

We study the optimization of full-duplex multi-input multioutput (MIMO) relays in a dual-hop multi-input single- output (MISO) link that is coupled with a weak direct end-toend connection, which we interpret as interference at the receiver. We design optimal power control, receive and transmit beamformers for the maximal performance of the end-to-end relay link. First, we derive the optimal transmit powers and receive beamformer. Next, we introduce a new figure of merit to approximate the optimal transmit beamformers based on the maximum signal-to-leakage-plus-noise ratio (SLNR) criterion. Finally, we design an iterative scheme to jointly optimize the end-to-end performance of the relay link.

\section{SYSTEM MODEL}

Let us consider a dual-hop link between a multi-antenna source node and a single-antenna destination node with a fullduplex MIMO relay connecting them, as depicted in Fig. 1. The source node transmits one data stream towards the relay node. The relay processes the received signal, and retransmits it towards the destination node using the same spectral resources; therefore the transmitted signal from the relay interferes at its receive side. The destination node receives the transmitted stream through two different links: the relay link and the direct source-destination link. The direct sourcedestination link is considered weak to be exploited in the destination. Yet, it causes interference at the destination because the derived signal is delayed due to the processing in the relay.

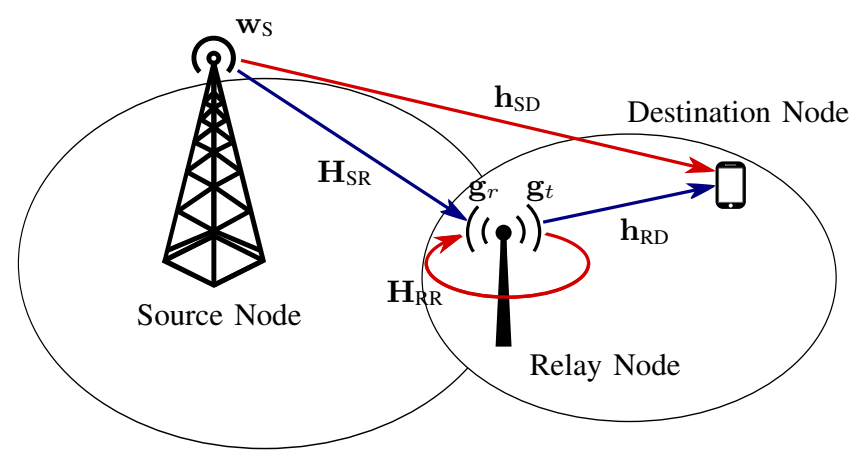

Fig. 1. A dual-hop MISO link with a full-duplex MIMO relay. 
We denote the number of antennas at the source node by $M_{\mathrm{S}}$, and the number of antennas at the receive and transmit sides of the relay by $N_{r}$ and $N_{t}$, respectively. In that case, the channel responses for the source-destination, source-relay, relay-destination links, and the receive-transmit link of the relay are denoted by $\mathbf{h}_{\mathrm{SD}} \in \mathbb{C}^{1 \times M_{\mathrm{s}}}, \mathbf{H}_{\mathrm{SR}} \in \mathbb{C}^{N_{r} \times M_{\mathrm{s}}}, \mathbf{h}_{\mathrm{RD}} \in$ $\mathbb{C}^{1 \times N_{t}}$, and $\mathbf{H}_{\mathrm{RR}} \in \mathbb{C}^{N_{t} \times N_{r}}$, respectively.

Due to the single-antenna destination node, the transmission is realized on a single stream. We denote by $\mathbf{w}_{\mathrm{S}} \in \mathbb{C}^{M_{\mathrm{S}} \times 1}$ the transmit beamformer at the source, and by $\mathbf{g}_{r} \in \mathbb{C}^{1 \times N_{r}}$ and $\mathbf{g}_{t} \in \mathbb{C}^{N_{t} \times 1}$ the receive and transmit beamformers at the relay, respectively. Let us also constrain the norm of the beamforming filters to one, i.e., $\left\|\mathbf{w}_{\mathrm{S}}\right\|=\left\|\mathbf{g}_{r}\right\|=\left\|\mathbf{g}_{t}\right\|=1$.

We denote the transmitted and received signals on the source-relay link by $x_{\mathrm{S}}$ and $y_{\mathrm{R}}$, as well as the transmitted and received signals on the relay-destination link by $x_{\mathrm{R}}$ and $y_{\mathrm{D}}$. Let us also denote the transmitted signals on the sourcedestination and relay-relay links by $\widetilde{x}_{\mathrm{S}}$ and $\widetilde{x}_{\mathrm{R}}$, respectively.

The transmitted signals on different links are modeled as independent and identically distributed, and assumed to be uncorrelated with each other due to large processing delay ${ }^{1}$ at the relay [3]; hence $\mathbb{E}\left\{x_{\mathrm{S}} x_{\mathrm{R}}\right\}=\mathbb{E}\left\{x_{\mathrm{S}} \widetilde{x}_{\mathrm{S}}\right\}=\mathbb{E}\left\{x_{\mathrm{R}} \widetilde{x}_{\mathrm{R}}\right\}=0$. The transmit powers at the source and relay are denoted by $p_{\mathrm{S}}$ and $p_{\mathrm{R}}$, respectively, i.e., $\mathbb{E}\left\{\left|x_{\mathrm{S}}\right|^{2}\right\}=\mathbb{E}\left\{\left|\widetilde{x}_{\mathrm{S}}\right|^{2}\right\}=p_{\mathrm{S}}$ and $\mathbb{E}\left\{\left|x_{\mathrm{R}}\right|^{2}\right\}=\mathbb{E}\left\{\left|\widetilde{x}_{\mathrm{R}}\right|^{2}\right\}=p_{\mathrm{R}}$, which are constrained by

$$
0<p_{\mathrm{S}} \leq P_{\mathrm{S}}, \quad 0 \leq p_{\mathrm{R}} \leq P_{\mathrm{R}}
$$

where $P_{\mathrm{S}}$ and $P_{\mathrm{R}}$ denote the maximum transmit powers at the source and relay.

The received signals, $y_{\mathrm{R}}$ and $y_{\mathrm{D}}$, are given by

$$
\begin{aligned}
& y_{\mathrm{R}}=\mathbf{g}_{r} \mathbf{H}_{\mathrm{SR}} \mathbf{w}_{\mathrm{S}} x_{\mathrm{S}}+\mathbf{g}_{r} \mathbf{H}_{\mathrm{RR}} \mathbf{g}_{t} \widetilde{x}_{\mathrm{R}}+\mathbf{g}_{r} \mathbf{n}_{\mathrm{R}} \\
& y_{\mathrm{D}}=\mathbf{h}_{\mathrm{RD}} \mathbf{g}_{t} x_{\mathrm{R}}+\mathbf{h}_{\mathrm{SD}} \mathbf{w}_{\mathrm{S}} \widetilde{x}_{\mathrm{S}}+n_{\mathrm{D}}
\end{aligned}
$$

where $\mathbf{n}_{\mathrm{R}} \in \mathbb{C}^{N_{r} \times 1}$ and $n_{\mathrm{D}} \in \mathbb{C}$ denote the additive noise at the relay and destination nodes, respectively.

The channel SNR expressions on the source-relay, $\gamma_{\mathrm{SR}}$, relay-destination, $\gamma_{\mathrm{RD}}$, relay-relay, $\gamma_{\mathrm{RR}}$, and sourcedestination, $\gamma_{\mathrm{SD}}$, links are given by

$$
\begin{aligned}
\gamma_{\mathrm{SR}}=\frac{\left|\mathbf{g}_{r} \mathbf{H}_{\mathrm{SR}} \mathbf{w}_{\mathrm{S}}\right|^{2}}{\sigma_{\mathrm{R}}^{2}}, & \gamma_{\mathrm{RD}}=\frac{\left|\mathbf{h}_{\mathrm{RD}} \mathbf{g}_{t}\right|^{2}}{\sigma_{\mathrm{D}}^{2}} \\
\gamma_{\mathrm{RR}}=\frac{\left|\mathbf{g}_{r} \mathbf{H}_{\mathrm{RR}} \mathbf{g}_{t}\right|^{2}}{\sigma_{\mathrm{R}}^{2}}, & \gamma_{\mathrm{SD}}=\frac{\left|\mathbf{h}_{\mathrm{SD}} \mathbf{w}_{\mathrm{S}}\right|^{2}}{\sigma_{\mathrm{D}}^{2}}
\end{aligned}
$$

where $\sigma_{\mathrm{R}}^{2}, \sigma_{\mathrm{D}}^{2}$ denote the noise powers at the relay and destination, i.e., $\mathbb{E}\left\{\mathbf{n}_{\mathrm{R}} \mathbf{n}_{\mathrm{R}}^{H}\right\}=\sigma_{\mathrm{R}}^{2} \mathbf{I}$ and $\mathbb{E}\left\{\left|n_{\mathrm{D}}\right|^{2}\right\}=\sigma_{\mathrm{D}}^{2}$.

The SINR expressions on the source-relay, $\gamma_{\mathrm{R}}$, and relaydestination, $\gamma_{\mathrm{D}}$, links are given by

$$
\gamma_{\mathrm{R}}=\frac{p_{\mathrm{S}} \gamma_{\mathrm{SR}}}{p_{\mathrm{R}} \gamma_{\mathrm{RR}}+1}, \quad \gamma_{\mathrm{D}}=\frac{p_{\mathrm{R}} \gamma_{\mathrm{RD}}}{p_{\mathrm{S}} \gamma_{\mathrm{SD}}+1}
$$

\footnotetext{
${ }^{1}$ Any processing delay that is longer than the transmission time of one symbol should suffice for both AF and DF.
}

The end-to-end SINR expressions under AF and DF protocols are given by

$$
\begin{aligned}
\gamma_{\mathrm{e} 2 \mathrm{e}}^{\mathrm{AF}} & =\frac{\gamma_{\mathrm{R}} \gamma_{\mathrm{D}}}{\gamma_{\mathrm{R}}+\gamma_{\mathrm{D}}+1} \\
\gamma_{\mathrm{e} 2 \mathrm{e}}^{\mathrm{DF}} & =\min \left\{\gamma_{\mathrm{R}}, \gamma_{\mathrm{D}}\right\}
\end{aligned}
$$

The maximum achievable data rate by the end-to-end relay link is given by $\log _{2}\left(1+\gamma_{\mathrm{e} 2 \mathrm{e}}\right)$ where $\gamma_{\mathrm{e} 2 \mathrm{e}}$ denotes the end-toend SINR.

\section{Transmit Power Allocations}

The transmit power allocation for the optimization of the end-to-end relay link is realized by the maximization of the end-to-end SINR for a fixed set of beamformers. First, we derive the optimal transmit powers at the source and relay separately with the assumption that the other transmit power is fixed. Next, we conclude that due to the duality of these power expressions, the optimal power allocation can be obtained by setting each of the derived transmit powers to its upper bound and picking the one yielding the higher end-to-end SINR.

\section{A. Under AF Relaying}

After taking the derivative of $\gamma_{\mathrm{e} 2 \mathrm{e}}^{\mathrm{AF}}$ in (7) with respect to $p_{\mathrm{S}}$, and setting it to zero, the optimal transmit power at the source is obtained as

$$
\hat{p}_{\mathrm{S}}=\min \left\{P_{\mathrm{S}}, \sqrt{\frac{\left(p_{\mathrm{R}} \gamma_{\mathrm{RD}}+1\right)\left(p_{\mathrm{R}} \gamma_{\mathrm{RR}}+1\right)}{\gamma_{\mathrm{SR}} \gamma_{\mathrm{SD}}}}\right\}
$$

Similarly, the optimal transmit power at the relay is obtained after taking the derivative of $\gamma_{\mathrm{e} 2 \mathrm{e}}^{\mathrm{AF}}$ with respect to $p_{\mathrm{R}}$, and setting it to zero by [4]

$$
\hat{p}_{\mathrm{R}}=\min \left\{P_{\mathrm{R}}, \sqrt{\frac{\left(p_{\mathrm{S}} \gamma_{\mathrm{SR}}+1\right)\left(p_{\mathrm{S}} \gamma_{\mathrm{SD}}+1\right)}{\gamma_{\mathrm{RR}} \gamma_{\mathrm{RD}}}}\right\}
$$

Due to the duality of (9) and (10), one can conclude that either $p_{\mathrm{S}}$ or $p_{\mathrm{R}}$ is always optimally equal to its maximum (1). Hence, the optimal power allocations are given by

$$
\left[p_{\mathrm{S}}, p_{\mathrm{R}}\right]= \begin{cases}{\left[P_{\mathrm{S}}, \hat{p}_{\mathrm{R}}\right],} & \left.\gamma_{\mathrm{e} 2 \mathrm{e}}^{\mathrm{AF}}\right|_{p_{\mathrm{R}}=P_{\mathrm{R}}} \leq\left.\gamma_{\mathrm{e} 2 \mathrm{e}}^{\mathrm{AF}}\right|_{p_{\mathrm{S}}=P_{\mathrm{S}}} \\ {\left[\hat{p}_{\mathrm{S}}, P_{\mathrm{R}}\right],} & \text { otherwise }\end{cases}
$$

\section{B. Under DF Relaying}

After setting $\gamma_{R}=\gamma_{D}$, the optimal transmit power at the source is obtained as

$$
\hat{p}_{\mathrm{S}}=\min \left\{P_{\mathrm{S}}, \frac{1}{2 \gamma_{\mathrm{SD}}}\left(\sqrt{1+\frac{4 \gamma_{\mathrm{SD}} \gamma_{\mathrm{RD}} p_{\mathrm{R}}\left[p_{\mathrm{R}} \gamma_{\mathrm{RR}}+1\right]}{\gamma_{\mathrm{SR}}}}-1\right)\right\}
$$

Similarly, the optimal $p_{R}$ is derived by setting $\gamma_{R}=\gamma_{D}$ and solving for $p_{\mathrm{R}}$ by [4]

$$
\hat{p}_{\mathrm{R}}=\min \left\{P_{\mathrm{R}}, \frac{1}{2 \gamma_{\mathrm{RR}}}\left(\sqrt{1+\frac{4 \gamma_{\mathrm{RR}} \gamma_{\mathrm{SR}} p_{\mathrm{S}}\left[p_{\mathrm{S}} \gamma_{\mathrm{SD}}+1\right]}{\gamma_{\mathrm{RD}}}}-1\right)\right\}
$$


Due to the duality between (12) and (13), the optimal pair of $p_{\mathrm{S}}$ and $p_{\mathrm{R}}$ is given by

$$
\left[p_{\mathrm{S}}, p_{\mathrm{R}}\right]= \begin{cases}{\left[P_{\mathrm{S}}, \hat{p}_{\mathrm{R}}\right],} & \left.\gamma_{\mathrm{e} 2 \mathrm{e}}^{\mathrm{DF}}\right|_{p_{\mathrm{R}}=P_{\mathrm{R}}} \leq\left.\gamma_{\mathrm{e} 2 \mathrm{e}}^{\mathrm{DF}}\right|_{p_{\mathrm{S}}=P_{\mathrm{S}}} \\ {\left[\hat{p}_{\mathrm{S}}, P_{\mathrm{R}}\right],} & \text { otherwise }\end{cases}
$$

\section{BEAMFORMER DESIGN}

In this section, we first derive the optimal receive beamformer based on the minimum MSE criterion with fixed transmit beamformers. Next, we adopt a new figure of merit to approximate the optimal transmit beamformers because the MMSE filtering is hard to derive due to the non-convexity of the end-to-end link. We define SLNR expressions for both source-relay and relay-destination links, which effectively decouple the end-to-end optimization problem of transmit beamformers into two parts: the maximization of the SLNR on the source-relay and relay-destination links. Finally, we present an iterative scheme to optimize the end-to-end relay link jointly by the minimum MSE and maximum SLNR-based beamformers as well as the optimal power control.

\section{A. By Minimum MSE}

When $\mathbf{w}_{\mathrm{S}}$ and $\mathbf{g}_{t}$ are fixed, the relay-destination link can be optimized by power control only, whereas the source-relay link is now exposed to a self-interference signal with a static beamforming direction. In that case, the optimal $\mathbf{g}_{r}$ is given by the MMSE filtering as

$$
\mathbf{g}_{r}=\frac{\mathbf{w}_{\mathrm{S}}^{H} \mathbf{H}_{\mathrm{SR}}^{H} \mathbf{R}_{\mathrm{I}}^{-1}}{\left\|\mathbf{w}_{\mathrm{S}}^{H} \mathbf{H}_{\mathrm{SR}}^{H} \mathbf{R}_{\mathrm{I}+\mathrm{N}}^{-1}\right\|}
$$

where $\mathbf{R}_{\mathrm{I}+\mathrm{N}}=p_{\mathrm{R}} \mathbf{H}_{\mathrm{RR}} \mathbf{g}_{t} \mathbf{g}_{t}^{H} \mathbf{H}_{\mathrm{RR}}^{H}+\sigma_{\mathrm{R}}^{2} \mathbf{I}$ is the autocorrelation of the interference-plus-noise signal.

However, (15) requires the optimal $p_{\mathrm{R}}$, whereas both (11) and (14) require the optimal $\mathbf{g}_{r}$. Because the transmit power expressions are non-convex, an iterative scheme using (15) does not guarantee convergence to global optimum. Therefore, we derive the optimal power allocations with the MMSE-based beamformer in closed forms below.

1) Power Allocations with MMSE-based Beamformer under $A F$ Relaying: After substituting $\mathbf{g}_{r}$ in (15) into (7), the optimal $p_{\mathrm{R}}[5]$ and $p_{\mathrm{S}}$ are obtained by

$$
\begin{aligned}
& \hat{p}_{\mathrm{S}}=\min \left\{P_{\mathrm{S}}, \frac{\alpha_{1}}{\alpha_{2}}\right\} \\
& \hat{p}_{\mathrm{R}}= \begin{cases}\varphi_{\mathrm{R}}, & \text { when } 0<\varphi_{\mathrm{R}} \leq P_{\mathrm{R}} \\
P_{\mathrm{R}}, & \text { otherwise }\end{cases}
\end{aligned}
$$

where $\alpha_{1}, \alpha_{2}, \varphi_{\mathrm{R}}$ are given by

$$
\begin{aligned}
& \alpha_{1}=\kappa_{1} p_{\mathrm{R}}^{2}+\kappa_{2} p_{\mathrm{R}}+\sigma_{\mathrm{R}}^{2} \frac{\kappa_{1}}{\kappa_{2}} p_{\mathrm{R}}+\sigma_{\mathrm{R}}^{2} \sigma_{\mathrm{D}}^{2} \\
& \alpha_{2}=\frac{1}{\sigma_{\mathrm{R}}^{2} \sigma_{\mathrm{D}}^{2}} \kappa_{5}\left(\kappa_{4}+\kappa_{3} p_{\mathrm{R}}\right) \\
& \varphi_{\mathrm{R}}=\frac{\sqrt{\beta_{1}^{2}+\beta_{2} \beta_{3}}+\beta_{1}}{\beta_{3}} \\
& \beta_{1}=\kappa_{2} \kappa_{3}\left(1+\frac{1}{\sigma_{\mathrm{R}}^{2} \sigma_{\mathrm{D}}^{2}}\left(\kappa_{4}+\kappa_{5}+\kappa_{4} \kappa_{5}\right) p_{\mathrm{S}}\right) p_{\mathrm{S}}
\end{aligned}
$$

$$
\begin{aligned}
\beta_{2}=\kappa_{2} \kappa_{4} & \left(1+\frac{1}{\sigma_{\mathrm{R}}^{2} \sigma_{\mathrm{D}}^{2}}\left(\kappa_{4}+\kappa_{5}+\kappa_{4} \kappa_{5}\right) p_{\mathrm{S}}\right) p_{\mathrm{S}} \\
\beta_{3}=\frac{1}{\sigma_{\mathrm{R}}^{2} \sigma_{\mathrm{D}}^{2}}( & \kappa_{1} \kappa_{2} \kappa_{4}-\kappa_{2} \kappa_{3}^{2} p_{\mathrm{S}}\left[\frac{1}{\sigma_{\mathrm{R}}^{2} \sigma_{\mathrm{D}}^{2}} \kappa_{5} p_{\mathrm{S}}+1\right] \\
& \left.\quad-\kappa_{2}^{2} \kappa_{3}-\kappa_{1} \kappa_{3} \kappa_{5} p_{\mathrm{S}}\right) p_{\mathrm{S}}-\frac{1}{\sigma_{\mathrm{D}}^{2}} \kappa_{1} \kappa_{3} p_{\mathrm{S}}
\end{aligned}
$$

for which the non-negative terms $\kappa_{1}, \kappa_{2}, \kappa_{3}, \kappa_{4}, \kappa_{5}$ are defined as

$$
\begin{aligned}
\kappa_{1} & =\left\|\mathbf{H}_{\mathrm{RR}} \mathbf{g}_{t}\right\|^{2}\left|\mathbf{h}_{\mathrm{RD}} \mathbf{g}_{t}\right|^{2} \\
\kappa_{2} & =\sigma_{\mathrm{R}}^{2}\left|\mathbf{h}_{\mathrm{RD}} \mathbf{g}_{t}\right|^{2} \\
\kappa_{3} & =\frac{\sigma_{\mathrm{D}}^{2}}{\sigma_{\mathrm{R}}^{2}}\left(\left\|\mathbf{H}_{\mathrm{SR}} \mathbf{w}_{\mathrm{S}}\right\|^{2}\left\|\mathbf{H}_{\mathrm{RR}} \mathbf{g}_{t}\right\|^{2}-\left|\mathbf{w}_{\mathrm{S}}^{H} \mathbf{H}_{\mathrm{SR}}^{H} \mathbf{H}_{\mathrm{RR}} \mathbf{g}_{t}\right|^{2}\right) \\
\kappa_{4} & =\sigma_{\mathrm{D}}^{2}\left\|\mathbf{H}_{\mathrm{SR}} \mathbf{w}_{\mathrm{S}}\right\|^{2} \\
\kappa_{5} & =\sigma_{\mathrm{R}}^{2}\left|\mathbf{h}_{\mathrm{SD}} \mathbf{w}_{\mathrm{S}}\right|^{2}
\end{aligned}
$$

The optimal pair of $p_{\mathrm{S}}$ and $p_{\mathrm{R}}$ can be obtained by the substitution of (16) and (17) into (11).

2) Power Allocations with MMSE-based Beamformer under DF Relaying: After substituting $\mathbf{g}_{r}$ in (15) into (8), the optimal $p_{\mathrm{R}}[5]$ and $p_{\mathrm{S}}$ are derived as

$$
\begin{aligned}
& \hat{p}_{\mathrm{S}}=\min \left\{P_{\mathrm{S}}, \frac{\sqrt{\tau_{1}^{2}+4 \tau_{2} \tau_{3}}-\tau_{1}}{2 \tau_{2}}\right\} \\
& \hat{p}_{\mathrm{R}}=\min \left\{P_{\mathrm{R}}, \frac{\sqrt{\varepsilon_{1}^{2}+4 \varepsilon_{2} \varepsilon_{3}}-\varepsilon_{1}}{2 \varepsilon_{2}}\right\}
\end{aligned}
$$

where $\tau_{1}, \tau_{2}, \tau_{3}, \varepsilon_{1}, \varepsilon_{2}, \varepsilon_{3}$ are given by

$$
\begin{aligned}
\tau_{1} & =\kappa_{4}+\kappa_{3} p_{\mathrm{R}} \\
\tau_{2} & =\frac{1}{\sigma_{\mathrm{R}}^{2} \sigma_{\mathrm{D}}^{2}} \kappa_{5}\left(\kappa_{4}+\kappa_{3} p_{\mathrm{R}}\right) \\
\tau_{3} & =\left(\kappa_{1} p_{\mathrm{R}}+\kappa_{2}\right) p_{\mathrm{R}} \\
\varepsilon_{1} & =\kappa_{2}-\kappa_{3}\left(\frac{1}{\sigma_{\mathrm{R}}^{2} \sigma_{\mathrm{D}}^{2}} \kappa_{5} p_{\mathrm{S}}+1\right) p_{\mathrm{S}} \\
\varepsilon_{2} & =\kappa_{1} \\
\varepsilon_{3} & =\kappa_{4}\left(\frac{1}{\sigma_{\mathrm{R}}^{2} \sigma_{\mathrm{D}}^{2}} \kappa_{5} p_{\mathrm{S}}+1\right) p_{\mathrm{S}}
\end{aligned}
$$

Similarly, the optimal pair of $p_{\mathrm{S}}, p_{\mathrm{R}}$ are obtained by substituting (29) and (30) into (14).

\section{B. By Maximum SLNR}

The leakage signal of a node is defined as the aggregate transmitted signal which is received and interpreted as interference by other nodes [6]. The SLNR expressions for the source-relay, $\widetilde{\gamma}_{R}$, and relay-destination, $\widetilde{\gamma}_{\mathrm{D}}$, links are given by

$$
\begin{aligned}
& \widetilde{\gamma}_{\mathrm{R}}=\frac{\left|\mathbf{g}_{r} \mathbf{H}_{\mathrm{SR}} \mathbf{w}_{\mathrm{S}}\right|^{2} p_{\mathrm{S}}}{\left|\mathbf{h}_{\mathrm{SD}} \mathbf{w}_{\mathrm{S}}\right|^{2} p_{\mathrm{S}}+\sigma_{\mathrm{R}}^{2}} \\
& \widetilde{\gamma}_{\mathrm{D}}=\frac{\left|\mathbf{h}_{\mathrm{RD}} \mathbf{g}_{t}\right|^{2} p_{\mathrm{R}}}{\left|\mathbf{g}_{r} \mathbf{H}_{\mathrm{RR}} \mathbf{g}_{t}\right|^{2} p_{\mathrm{R}}+\sigma_{\mathrm{D}}^{2}}
\end{aligned}
$$


In that case, the optimal $\mathbf{w}_{\mathrm{S}}$ and $\mathbf{g}_{t}$ maximizing SLNR are given by

$$
\begin{aligned}
\mathbf{w}_{\mathrm{S}} & =\left(p_{\mathrm{S}} \mathbf{h}_{\mathrm{SD}}^{H} \mathbf{h}_{\mathrm{SD}}+\sigma_{\mathrm{R}}^{2} \mathbf{I}\right)^{-1} \mathbf{H}_{\mathrm{SR}}^{H} \mathbf{g}_{r}^{H} \\
\mathbf{g}_{t} & =\left(p_{\mathrm{R}} \mathbf{H}_{\mathrm{RR}}^{H} \mathbf{g}_{r}^{H} \mathbf{g}_{r} \mathbf{H}_{\mathrm{RR}}+\sigma_{\mathrm{D}}^{2} \mathbf{I}\right)^{-1} \mathbf{h}_{\mathrm{RD}}^{H}
\end{aligned}
$$

Notice that (39) and (40) does not necessarily maximize the end-to-end SINR. However, one can show that the SLNRbased transmit beamformers are guaranteed to yield the maximum data rate on the condition that at least one of the following equalities hold.

$$
p_{\mathrm{S}} \gamma_{\mathrm{SR}}=p_{\mathrm{R}} \gamma_{\mathrm{RD}}, \quad p_{\mathrm{S}} \gamma_{\mathrm{SD}}=p_{\mathrm{R}} \gamma_{\mathrm{RR}}
$$

where we assume $\sigma_{\mathrm{R}}^{2}=\sigma_{\mathrm{D}}^{2}$.

\section{By Joint Minimum MSE and Maximum SLNR}

The optimization of the end-to-end transmission can be achieved by a set of joint iterations between the MMSEbased receive beamformer with optimal transmit powers and the maximum SLNR-based transmit beamformers.

The iterative scheme can be initiated by setting $\mathbf{w}_{\mathrm{S}}^{(0)}$ as the right singular vector of $\mathbf{H}_{\mathrm{SR}}$ that corresponds to the largest singular value, and $\mathbf{g}_{t}^{(0)}$ as the hermitian transpose of $\mathbf{h}_{\mathrm{RD}}$. The optimal $\mathbf{g}_{r}^{(i)}, p_{\mathrm{S}}^{(i)}$, and $p_{\mathrm{R}}^{(i)}$ for the $i^{\text {th }}$ iteration are calculated by (15), (11), (14), where $\hat{p}_{\mathrm{S}}$ and $\hat{p}_{\mathrm{R}}$ are given by either (16), (17) or (29), (30). Next, the optimal $\mathbf{w}_{\mathbf{S}}^{(i)}$ and $\mathbf{g}_{t}^{(i)}$ are approximated by (39) and (40). One should notice that the convergence is not always guaranteed due to the non-convexity of the transmit power expressions. Hence, the algorithm is terminated when the current end-to-end SINR does not increase any more. Additionally, the algorithm can also be terminated when a certain number of iterations is exceeded, i.e., $i>\mathcal{I}$, where $\mathcal{I}$ is a large number. In our simulations, we choose $\mathcal{I}=100$. The joint iterative algorithm is presented in Table $\mathrm{I}$.

\section{Numerical ANALysis}

The following simulation results demonstrate the performance analysis of the proposed joint iterative scheme.

The MIMO relay is equipped with two transmit $\left(N_{t}=2\right)$ and two receive antennas $\left(N_{r}=2\right)$, whereas the source employs four antennas $\left(M_{\mathrm{S}}=4\right)$. All the channels are modeled to experience flat fading with the channel elements being independent identically distributed complex Gaussian random variables. The average gain of each channel element is modeled as $15 \mathrm{~dB}$ for the source-relay and relay-destination channels, whereas each element of the relay-relay and sourcedestination interference channels are modeled to have the same average gain, which is varied from $0 \mathrm{~dB}$ to $10 \mathrm{~dB}$. The additive noise terms at the relay and destination are modeled as Gaussian with zero-mean and unit-variance. The maximum transmit powers at the source and relay are defined as one.

Fig. 2 shows the achievable data rates by the joint iterative scheme with the SLNR-based transmit beamformers, and compares them to the performance of the conventional maximum ratio transmit (MRT) and zero-forcing (ZF) transmit
TABLE I

ITERATIVE OPTIMIZATION OF THE END-TO-END TRANSMISSION

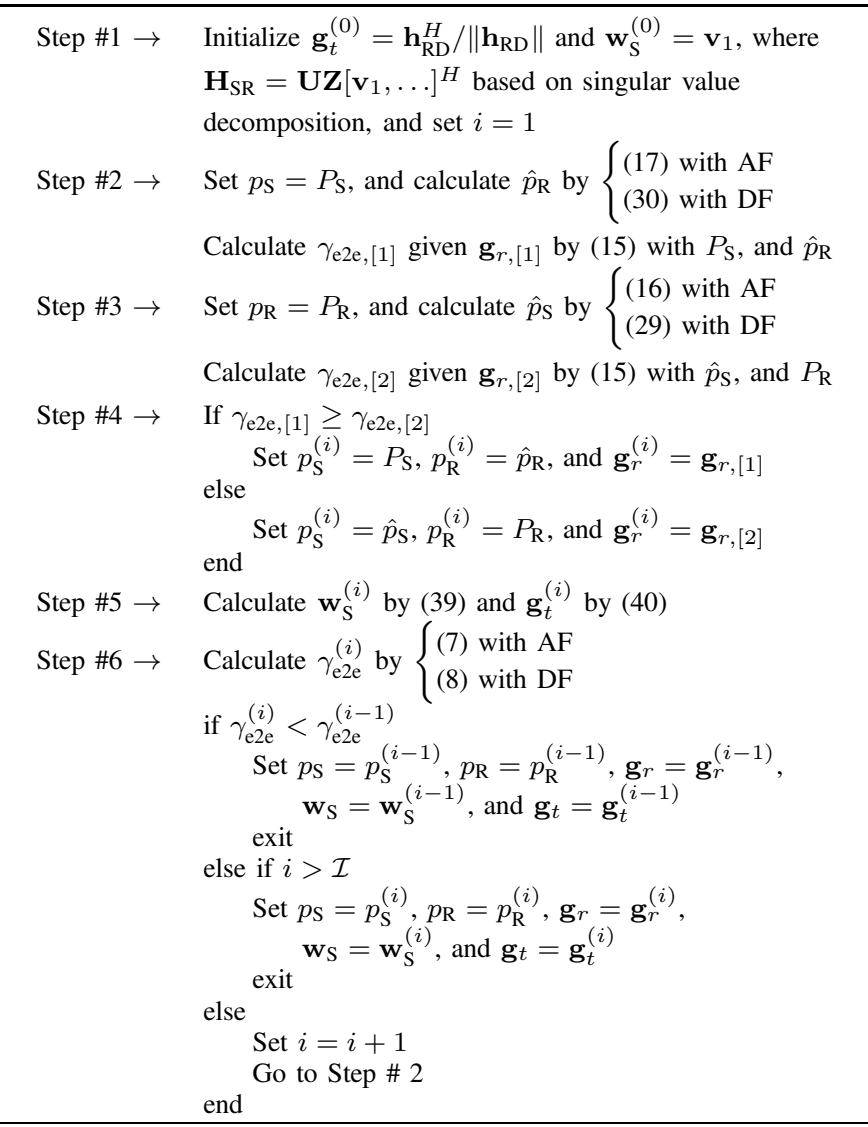

beamformers as well as that of direct-link transmission. The MRT beamformer at the source is given by the right singular vector of $\mathbf{H}_{\mathrm{SR}}$ that corresponds to the largest singular value, whereas the MRT beamformer at the relay is given by the normalized hermitian transpose of $\mathbf{h}_{\mathrm{RD}}$. The $\mathrm{ZF}$ transmit beamformer at the source is given by the projection transform of the right singular vector of $\mathbf{H}_{\mathrm{SR}}$ corresponding to the largest singular value to the nullspace of $\mathbf{h}_{\mathrm{SD}}$, whereas the $\mathrm{ZF}$ transmit beamformer at the relay is given by the projection transform of $\mathbf{h}_{\mathrm{RD}}^{H}$ to the nullspace of $\mathbf{g}_{r} \mathbf{H}_{\mathrm{RR}}$ where $\mathbf{g}_{r}$ is chosen as the left singular vector of $\mathbf{H}_{\mathrm{SR}}$ that corresponds to the largest singular value. Naturally, the direct-link transmission is realized by the MRT beamformer at the source with maximum transmit power, i.e., $p_{\mathrm{S}}=P_{\mathrm{S}}$, while the relay node is turned off by $p_{\mathrm{R}}=0$.

Note that all the schemes in Fig. 2 use the optimal MMSEbased receive beamformer and transmit powers. The vertical axis represents the achievable data rates, whereas the horizontal axis represents the average gain of the relay-relay and source-destination interference channels. The solid lines show the achievable rates with DF relaying, whereas the dashed lines represent the data rates with $\mathrm{AF}$ relaying. We observe that the joint iterative scheme outperforms the conventional transmit beamformers with both AF and DF relaying. The MRT beamformer severely suffers from high interference levels, whereas the ZF beamformer is insensitive to the effect of interference. 


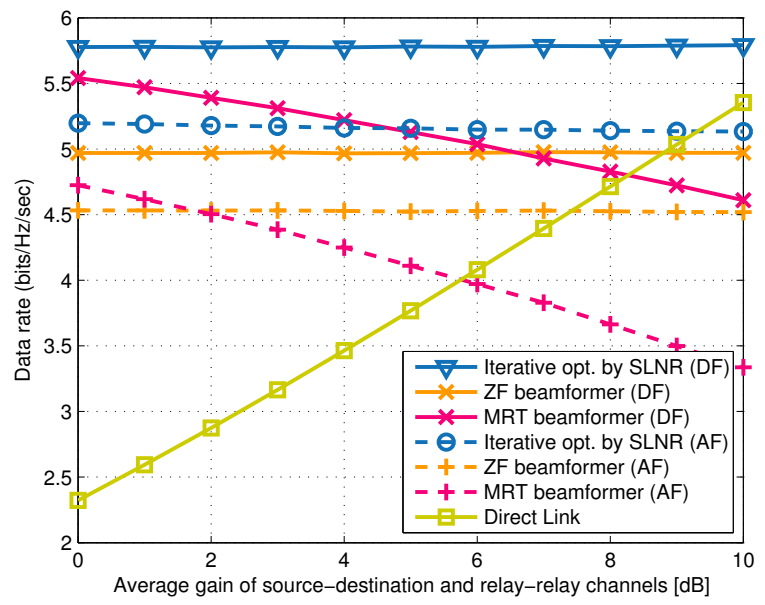

Fig. 2. The achievable data rates by the joint iterative algorithm vs. the conventional transmit beamformers.

The direct-link transmission performs better while the gain of the source-destination channel improves. When the gains of the source-destination and relay-relay channels are $10 \mathrm{~dB}$, the direct link achieves higher data rate than the proposed SLNRbased scheme with AF relaying. However, the relay link is still preferable over the direct link under the DF protocol in terms of higher data rate.

In order to evaluate the contribution of the SLNR-based transmit beamformers alone within the joint iterative algorithm, the MRT-based and ZF-based transmit beamformers are integrated into the algorithm as reference schemes. In particular, Step \#5 of Table I is replaced by the MRT and ZF beamformers. Fig. 3 shows the achievable rates by the joint iterative algorithm with the SLNR-based, MRT-based, and ZFbased transmit beamformers. We observe that the SLNR-based beamformer outperforms the conventional beamformers. Furthermore, both the MRT and ZF-based beamformers achieve higher data rates under low and high interference levels relative to those in Fig. 2.

In contrast to the results in Fig. 2, the ZF-based beamformer achieves higher data rates with higher interference levels in Fig. 3. This counterintuitive phenomenon can be explained as follows. When the interference levels are low, the interplay between the maximization of the useful signal and the minimization of the interference signal gains is a delicate procedure, hence the iterations start to bounce between them forcing the algorithm to terminate faster. In contrast, when the interference levels are high, the interplay is a cruder procedure, therefore the first drop in the data rate happens relatively slower letting the algorithm run for more iterations. As a result, the iterative joint optimization with the $\mathrm{ZF}$ beamformer achieves higher data rates under high interference.

\section{CONCLUSIONS}

We studied the optimization of a dual-hop multi-input single-output link with a full-duplex multi-input multi-output

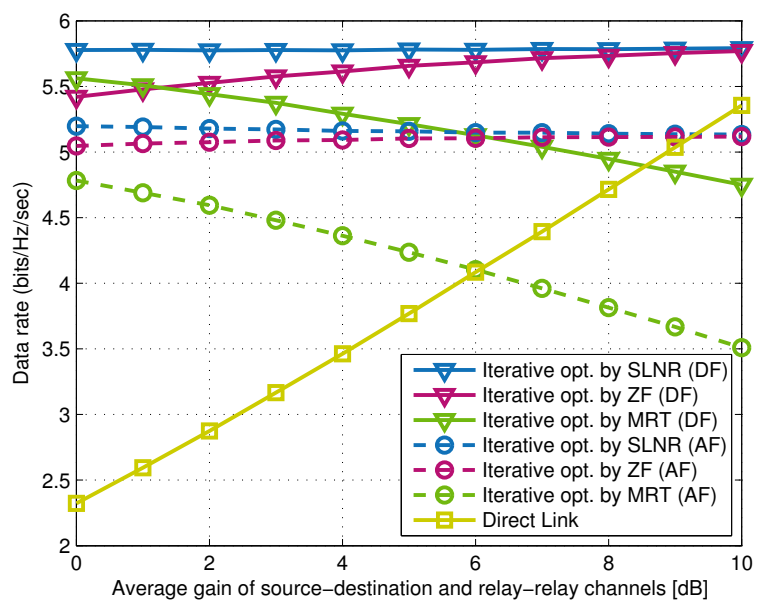

Fig. 3. The achievable data rates by the joint iterative algorithm with SLNR, MRT, and ZF-based transmit beamformers.

relay with both the amplify-and-forward and the decodeand-forward protocols, where the direct end-to-end link is interpreted as interference at the destination. We designed transmit power control and beamforming filters to optimize the end-to-end transmission. First, we combined the optimal minimum mean square error-based receive beamformer with the optimal transmit powers to tackle the non-convexity problem inherent to an iterative approach. Next, we introduced the signal-to-leakage-plus-noise ratio as a new figure of merit to approximate the optimal transmit beamformers at the source and relay. In addition, we designed an iterative algorithm to jointly optimize the receive and transmit beamformers. Our numerical analysis shows that the proposed joint iterative scheme with the leakage-based transmit beamformers outperforms the conventional beamforming methods at both low and high interference levels.

\section{REFERENCES}

[1] D. W. Bliss, P. A. Parker, and A. R. Margetts, "Simultaneous transmission and reception for improved wireless network performance," in Proc. IEEE/SP 14th Workshop on Statistical Signal Processing, Madison, WI, USA, August 2007, pp. 478-482.

[2] K. Haneda, E. Kahra, S. Wyne, C. Icheln, and P. Vainikainen, "Measurement of loop-back interference channels for outdoor-to-indoor fullduplex radio relays," in Proc. 4th European Conference on Antennas and Propagation, Barcelona, Spain, April 2010, pp. 1-5.

[3] R. Hu, C. Hu, J. Jiang, X. Xie, and L. Song, "Full-duplex mode in amplify-and-forward relay channels: Outage probability and ergodic capacity," International Journal of Antennas and Propagation, vol. 2014, February 2014. [Online]. Available: http://dx.doi.org/10.1155/2014/347540

[4] T. Riihonen, S. Werner, and R. Wichman, "Hybrid full-duplex/half-duplex relaying with transmit power adaptation," IEEE Transactions on Wireless Communications, vol. 10, no. 9, pp. 3074-3085, September 2011.

[5] U. Ugurlu, T. Riihonen, and R. Wichman, "Optimized in-band full-duplex MIMO relay in single-stream transmission," February 2014, manuscript submitted for publication.

[6] A. Tarighat, M. Sadek, and A. H. Sayed, "A multi user beamforming scheme for downlink MIMO channels based on maximizing signal-toleakage ratios," in Proc. IEEE International Conference on Acoustics, Speech, and Signal Processing, vol. 3, March 2005, pp. 1129-1132. 